\title{
One-year evaluation of epiduroscopy in chronic back pain with and without radiculopathy: a retrospective study
}

\author{
Mert Akbas ${ }^{1}$, Huseyin Babun², Haitham Hamdy Salem ${ }^{3^{*}}$ (D) Tamer Hussien Emara ${ }^{3}$, Shereen Elmosly ${ }^{4}$, \\ Emel Gunduz ${ }^{2}$, Bilge Karsli ${ }^{1}$ and Fatih Asik ${ }^{2}$
}

\begin{abstract}
Background: Back pain is a frequent complaint among the individuals in the society. It significantly affects the daily activities and the social and psychological life aspects leading to an economic health burden. Epiduroscopy is a relatively new minimally invasive technique that is used as a diagnostic and therapeutic tool in cases of chronic back pain.
\end{abstract}

Aim of the study: To evaluate the extent of long-term benefit of epiduroscopy in patients with refractory chronic back pain with/without radiculopathy.

Materials and methods: Retrospective data of 148 patients with failed back surgery syndrome (FBSS) and/or symptomatic lumbosacral disc prolapse who underwent epiduroscopy were collected. A 50\% reduction in the visual analog scale (VAS) score was set as the primary outcome. Pre- and post-procedure analgesic use, quality of sleep, and changes in the activities of daily livings (ADLs) after 1-year follow-up were reviewed. Incidence and types of complications were recorded.

Results: The mean age of the studied sample was 56.6 years with a higher percentage of females (61.5\%). Patients having radicular pain represented $45.1 \%$. Disc prolapse was prevalent (61.5\%) compared to patients with FBSS (38.5\%). A reduction of 50\% or more in VAS score was reached in $52.7 \%(p<0.01)$. This reduction was more evident in younger patients $(p=0.004)$. There was an improvement in ADLs $(p<0.01)$, quality of sleep $(p<0.05)$, and analgesic intake $(p<0.05)$.

Conclusion: Epiduroscopy is a relatively recent, safe, and minimally invasive tool that showed effectiveness in the difficult to treat patients with back pain with/without radiculopathy especially in FBSS.

Keywords: Epiduroscopy, Chronic back pain, Radiculopathy, Disc prolapse, Fail back surgery syndrome

\section{Introduction}

The majority of individuals in the society (70-90\%) suffered from back pain at least one time at any period of their life [1]. Back pain is one of the most important reasons for labor loss. It significantly affects the daily life activities and the social and psychological personal aspects thus ending in an inflation in the health expenses across the world [2, 3].

Epiduroscopy is a new minimally invasive technique that is used as a diagnostic and therapeutic tool in cases

\footnotetext{
* Correspondence: haithamhamdy@yahoo.com

${ }^{3}$ Department of Neurology, Ain Shams University, Cairo, Egypt

Full list of author information is available at the end of the article
}

of chronic back pain with/without radiculopathy $[4,5]$. It enables understanding the reason of pain, increasing the quality and effectiveness of drug injection and lysis of fibrotic areas when required $[1,6]$.

In this study, we aim to evaluate the extent of longterm benefit of epiduroscopy in patients with refractory chronic back pain with/without radiculopathy.

\section{Material and methods}

We retrospectively analyzed data of 148 patients with low back pain due to failed back surgery syndrome (FBSS) or symptomatic lumbosacral disc prolapse who 
underwent epiduroscopy in Akdeniz University, Antalya, Turkey, between January 2015 and May 2017.

All patients were older than 18 years, with persistent low back pain with/without radiculopathy not responding to conventional medical and physical treatment for a minimum of 6 months. Exclusion criteria included pregnant and lactating ladies, patients with organ failure, and presence of other causes of back pain.

Epiduroscopy procedure was performed under local anesthesia and light sedation with fluoroscopic guidance. The patient was placed in the prone position, sterilized, and then an $18 \mathrm{G}$ needle is introduced into the epidural space through the sacral hiatus. A fiber optic endoscope (POLYDIAGNOST GmbH Schamerstrasse, Germany) is introduced into the epidural space and directed cephalically to the target level (Fig. 1). The epidural space was examined for the presence of perineural adhesions, root

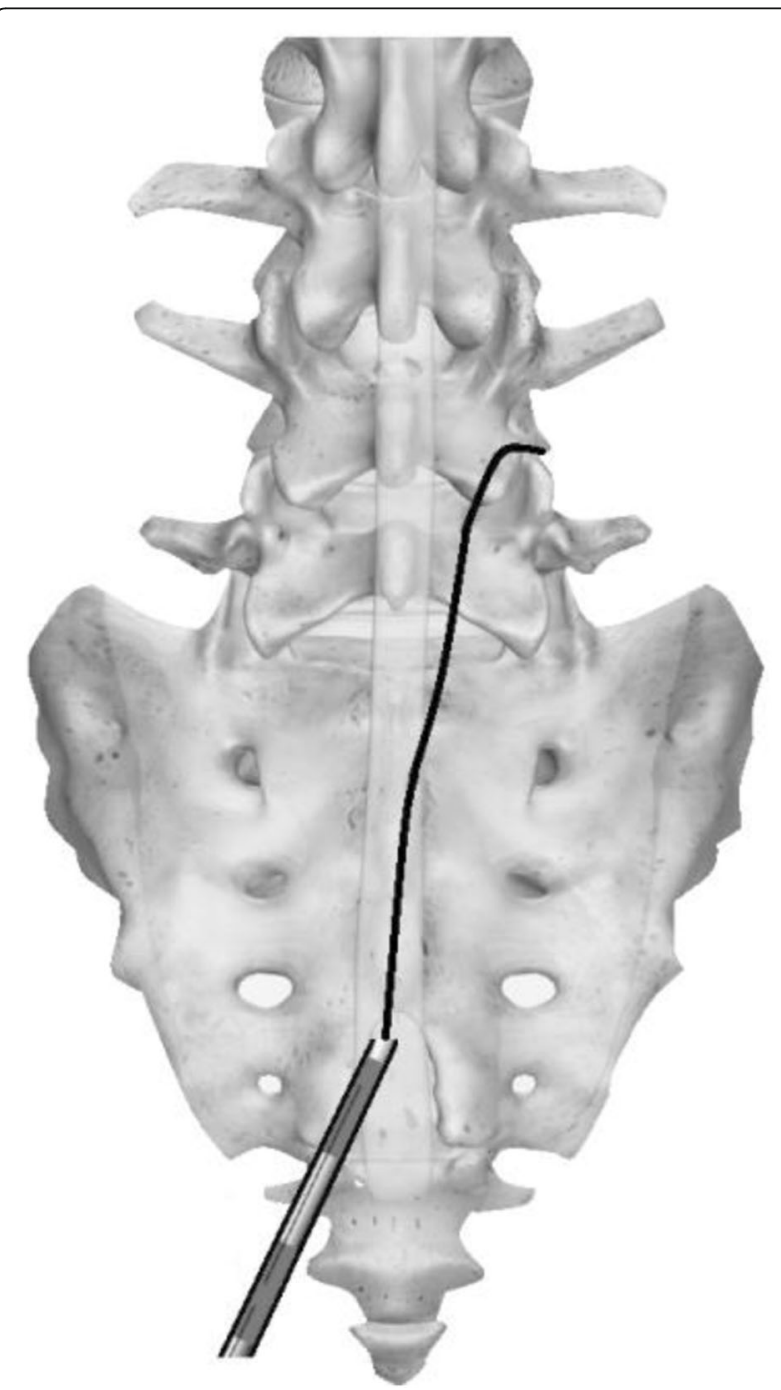

Fig. 1 Fiber optic endoscope is introduced into the epidural space and directed cephalically to the target level compression, congestion, and ligamentum flavum thickening. Mechanical motion and hydrolysis $(70 \mathrm{cc}$ normal saline, 5 cc $2 \%$ xylocaine, and $8 \mathrm{mg}$ dexamethasone) were used for lysis of adhesions and root compression release under guided video monitoring. Following the procedures, patients were monitored for the next $3 \mathrm{~h}$.

A reduction of $50 \%$ in the visual analog scale (VAS) score was set as the primary outcome. Pre- and postprocedure analgesic use, quality of sleep, and changes in the activities of daily livings (ADLs) using 3-point Likert scale after 1-year follow-up were reviewed from the patients' records. Incidence and types of complications were recorded.

\section{Ethical issues}

Patients' consents for epiduroscopy intervention were reviewed. Patient evaluation was performed during the post-procedure routine policlinic follow-up. The study was approved by the ethical committee of Akdeniz University on November 4, 2018 (no. 263).

\section{Statistical analysis}

Data were coded into Microsoft Access (Windows 10); then, data analysis was performed using the Statistical Package of Social Science (SPSS) version 22 (SPSS Inc., Chicago, IL). Simple descriptive analysis in the form of numbers and percentages was used for qualitative data, and arithmetic means as central tendency measurement and standard deviations as measure of dispersion were applied for quantitative parametric data. Quantitative data included in the study was first tested for normality by One-Sample Kolmogorov-Smirnov test in each study group then inferential statistic tests were selected. Oneway analysis of variance (ANOVA) test was used to compare more than two independent variables (continuous variables) of quantitative data. Bivariate Pearson correlation test $\left(\chi^{2}\right.$ test) was used to test the association between dichotomous variables. A $p$ value $\leq 0.05$ was considered the cutoff value for significance.

\section{Results}

A total of 148 patients were included in the study; $61.5 \%$ $(n=91)$ were females and $38.5 \%(n=57)$ were males. Mean age of the studied sample was $56.6( \pm 13.4)$. All patients suffered from back pain with a mean duration of $5.8( \pm 4.6)$ years. Patients complaining of radicular pain were $68(45.1 \%)$. Disc prolapse was prevalent in 91 (61.5\%) patients compared to $57(38.5 \%)$ patients with FBSS.

Among the studied sample, $52.7 \%(n=78)$ of patients achieved a reduction of $50 \%$ or more in VAS score with a mean reduction of $4( \pm 3.1)$ points. Other outcome measures showed significant improvement after 1-year duration (Table 1). 
Table 1 Outcome measures before and after epiduroscopy

\begin{tabular}{llll}
\hline & Baseline & After 1 year & $p$ value \\
\hline VAS score & $9.1 \pm 0.9$ & $5.1 \pm 3.1$ & $<0.01$ \\
ADLs & & & \\
$\quad$ Not affected & $0(0.0 \%)$ & $39(26.4 \%)$ & $<0.01$ \\
Moderately affected & $24(16.2 \%)$ & $58(39.2 \%)$ & \\
$\quad$ Severely affected & $124(83.8 \%)$ & $51(34.5 \%)$ & \\
Quality of sleep & & & \\
Not affected & $18(12.2 \%)$ & $32(21.6 \%)$ & $<0.05$ \\
Moderately affected & $57(38.5 \%)$ & $54(36.5 \%)$ & \\
$\quad$ Severely affected & $73(49.3 \%)$ & $62(41.9 \%)$ & \\
Use of analgesics & & & $<0.05$ \\
No & $0(0.0 \%)$ & $46(31.1 \%)$ & \\
Yes & $148(100 \%)$ & $102(68.9 \%)$ & \\
\hline
\end{tabular}

VAS visual analog scale, $A D L$ activity of daily living

Upon comparing patients with FBSS and disc prolapse, both showed improvement in pain scores after 1-year duration $(p>0.05)$ (Fig. 2). Our results showed that 37/57 (65\%) of patients with FBSS had $50 \%$ or more reduction in VAS score compared to $41 / 91$ (45.1\%) of patients with disc prolapse $(p=0.03)$ (Fig. 3).

Patients who experienced $50 \%$ or more reduction in pain scores were significantly younger than those who did not $[53.7( \pm 13.1)$ vs $60( \pm 13) ; p=0.004]$. However, there was no difference in improvement regarding sex as $57.7 \%$ of females and $42.3 \%$ of males showed $50 \%$ or more reduction in pain score $(p=0.3)$. Similarly, duration of illness showed no correlation with improvement ( $p=0.08$ ).

Only 4 (2.7\%) patients developed complications during the epiduroscopy procedure. One patient had dural puncture, and the endoscope was removed and reinserted again in the epidural space. Two patients complained of post-procedural numbness in lower limbs, and 1 patient developed headache. All complications were self-limiting and resolved within the post-procedural monitoring period. During the 1-year follow up, only 17 (11.5\%) patients of the studied population experienced persistent pain and subsequently underwent surgical interventions to relieve pain after the failure of epiduroscopy.

\section{Discussion}

Epiduroscopy is a relatively new technique. Several studies showed that it is a safe and effective modality in the treatment of patients with chronic back pain with/without radiculopathy [7-9]. We investigated the long-term efficacy of epiduroscopy in a large sample of patients with FBSS and disc prolapse.

Our results showed a sustained $50 \%$ or more improvement in pain scores (52.3\% of the studied sample). This result is in concordance with several other studies with variable follow-up durations ranging from 3 months to maximum of 5 years [10-13].

Insomnia is a known important complaint in patients with chronic pain [63]. Our results showed that sleep quality improved significantly after epiduroscopy and this can be explained by the reciprocal relationship between pain and sleep quality [14]. Similarly, ADLs improved after the procedure. These positive changes in sleep quality and ADLs may help patients to regain their social and vocational rules [15-17].

The average age of the patients included in our study was 56.6 years which is similar to many previous studies $[4,18,19]$. In the studied population, younger patients showed better improvement regarding VAS score. This

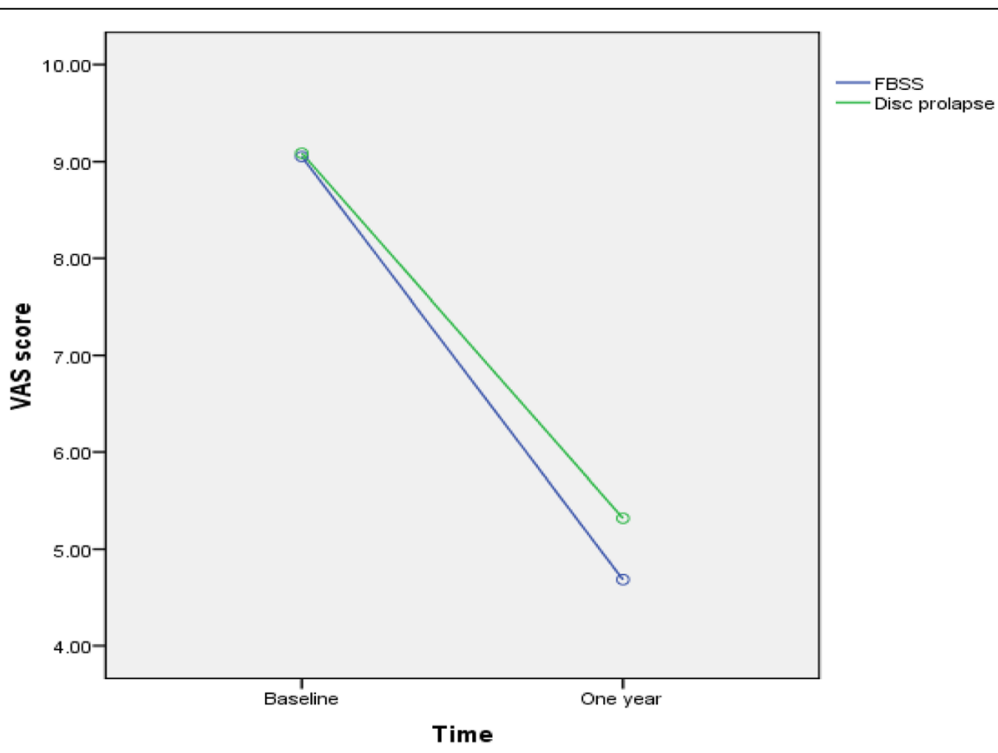

Fig. 2 Improvement in VAS score over time in patients with FBSS and disc prolapse. VAS, visual analog scale; FBSS, failed back surgery syndrome 


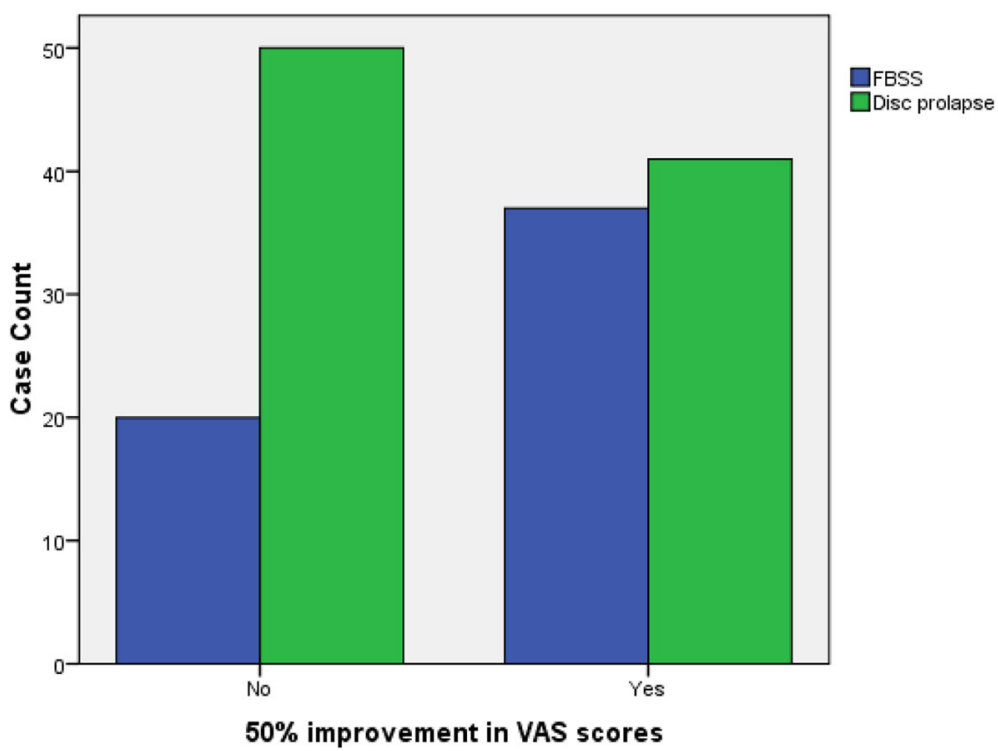

Fig. 3 A 50\% or more reduction in VAS score over time in patients with FBSS and disc prolapse. VAS, visual analog scale; FBSS, failed back surgery syndrome

can be attributed to decreased physical activities and increase in spinal canal stenosis in elder patients.

Both patients with FBSS and disc herniation improved in pain scores after epiduroscopy [9, 10, 12, 19-21]. Interestingly, in our study sample, although both groups of patients improved, FBSS patients achieved significantly higher rates of $50 \%$ or more reduction in VAS score $(65 \%)$ compared to patients with disc prolapse (45.1\%). This could be explained by the mechanical adhesiolysis effect of epiduroscopy that is considered the main cause of pain in FBSS. In cases with lumbosacral disc prolapse, steroids administered during the procedure help cleanse the inflammatory agents in the epidural space which is considered the main cause of pain [22].

Our rate of complications was $2.7 \%$, and all were selflimiting. This highlights the safety of this procedure that was previously shown in other studies [23]. Our study is limited by being retrospective. Longer follow-up beyond 1 -year duration is recommended to prove the sustainability of improvement after epiduroscopy. Long-term prospective multi-centric studies will prove the efficacy of epiduroscopy as a standard treatment modality.

\section{Conclusion}

Epiduroscopy is a relatively new, safe, and minimally invasive technique that showed a good and sustained response in the difficult to treat patients with back pain with/without radiculopathy specially patients with FBSS.

Acknowledgements

Not applicable

\section{Authors' contributions}

MA participated in the design of the study, performed some of the procedures for epiduroscopy, and revised the manuscript. HB collected the data and participated in data analysis. HHS performed some of the procedures for epiduroscopy and participated in manuscript production. THE participated in data analysis and manuscript production. SM participated in the data analysis and manuscript production. EG collected the data and participated in the data analysis. BK collected the data and participated in the data analysis. FA analyzed the data. All authors read and approved the final manuscript.

\section{Funding}

None

\section{Availability of data and materials}

Available on request

\section{Ethics approval and consent to participate}

The study was approved by the ethical committee of Akdeniz University on 11.04.2018 (No. 263). Patients' consents for epiduroscopy intervention were reviewed.

\section{Consent for publication}

Not applicable

\section{Competing interests}

The authors declare that they have no competing interests.

\section{Author details}

${ }^{1}$ Department of Pain Medicine, Akdeniz University, Antalya, Turkey.

${ }^{2}$ Department of Anesthesiology, Akdeniz University, Antalya, Turkey.

${ }^{3}$ Department of Neurology, Ain Shams University, Cairo, Egypt. ${ }^{4}$ Department

of Neurology, Fayoum University, Fayoum, Egypt.

Received: 5 November 2019 Accepted: 20 December 2019

Published online: 31 December 2019

\section{References}

1. Erdine S. Ağrı. Istanbul: Nobel Kitabevi; 1987.

2. Morgan G, Mikhail M, Murray M, Tulunay M, Cuhruk H. Klinik anesteziyoloji. Ankara: Güneş Tip Kitabevleri; 2008. 
3. Weiner D, Sakamoto S, Perera S, Breuer P. Chronic low back pain in older adults: prevalence, reliability, and validity of physical examination findings. JAGS. 2006;54(1):11-20.

4. Avellanal M, Diaz-Reganon G. Interlaminar approach for epiduroscopy in patients with failed back surgery syndrome. Br J Anaesth. 2008;101 (2):244-9.

5. Avellanal M, Diaz-Reganon G, Orts A, Soto S. One-year results of an algorithmic approach to managing failed back surgery syndrome. Pain Res Manag. 2014;19(6):313-6.

6. Dashfield A, Taylor M, Cleaver J, Farrow D. Comparison of caudal steroid epidural with targeted steroid placement during spinal endoscopy for chronic sciatica: a prospective, randomized, double-blind trial. Br J Anaesth. 2005;94(4):514-9.

7. Conn A, Buenaventura RM, Datta S, Abdi S, Diwan S. Systematic review of caudal epidural injections in the management of chronic low back pain. Pain Physician. 2009;12(1):109-35.

8. Geurts J, Kallewaard J, Richardson J, Groen G. Targeted methylprednisolone acetate/hyaluronidase/clonidine injection after diagnostic epiduroscopy for chronic sciatica. Reg Anesth Pain Med. 2002;27(4):343-52.

9. Richardson J, McGurgan P, Cheema S, Prasad R, Gupta S. Spinal endoscopy in chronic low back pain with radiculopathy a prospective case series. Anaesthesia. 2001;56(5):454-60.

10. Manchikanti L, Boswell M, Rivera J, Pampati V, Damron K, McManus C, et al. A randomized, controlled trial of spinal endoscopic adhesiolysis in chronic refractory low back and lower extremity pain. BMC Anesthesiol. 2005;5:1.

11. Di Donato A, Fontana C, Alemanno D, Di Giacomo A. Epiduroscopy in treatment of degenerative chronic low back pain: a prospective analysis and follow-up at 60 months. Clin Res Regul Aff. 2010;27(3):69-74.

12. Di Donato A, Fontana C, Pinto R, Beltrutti D, Pinto G. The effectiveness of endoscopic epidurolysis in treatment of degenerative chronic low back pain: a prospective analysis and follow-up at 48 months. Acta Neurchir. 2011;108:67-73

13. Agalhaes FNO, Soares SC, Torres JM, et al. Effects of ozone applied by spinal endoscopy in patients with chronic pain related to failed back surgery syndrome: a pilot study. Neuropsychiatr Dis Treat. 2013;9:1759-66.

14. Bohra MH, Kaushik C, Temple D, Chung SA, Shapiro CM. Weighing the balance: how analgesics used in chronic pain influence sleep? Br. J. Pain. 2014:8(3):107-18. https://doi.org/10.1177/2049463714525355.

15. Von Spannenberg SS, Jones GT, Macfarlane GJ. The evidence base for managing older persons with low back pain. Br J Pain. 2012;6(4):166-9.

16. Taylor RS, Taylor RJ. The economic impact of failed back surgery syndrome. Br J Pain. 2012;6(4):174-81.

17. Tharmanathan P, Adamson J, Ashby R, Eldabe S. Diagnosis and treatment of failed back surgery syndrome in the UK: mapping of practice using a crosssectional survey. Br J Pain. 2012;6(4):142-52.

18. Manchikanti L, Rivera J, Pampati VS, Damron KS, Beyer CD, Brandon DE, et al. Spinal endoscopic adhesiolysis in the management of chronic low back pain: a preliminary report of a randomized, double-blind trial. Pain Physician. 2003;6:259-68

19. Igarashi T. Lysis of adhesions and epidural injection of steroid/local anaesthetic during epiduroscopy potentially alleviate low back and leg pain in elderly patients with lumbar spinal stenosis. Br J Anaesth. 2004;93(2):181-7.

20. Helm S, Hayek SM, Colson J, Chopra P, Deer TR, Justiz R, Hameed M, Falco FJ. Spinal endoscopic adhesiolysis PLSS. Pain Physician. 2013;16(2 Suppl).

21. Abdulla A, Adams N, Bone M, Elliott A, Gaffin J, Jones D, et al. Evidencebased clinical practice guidelines on the management of pain in older people: executive summary. Br J Pain. 2013;7(3):152-4.

22. Hazer DB, Acarbas A, Rosberg HE. The outcome of epiduroscopy treatment in patients with chronic low back pain and radicular pain, operated or nonoperated for lumbar disc herniation: a retrospective study in 88 patients. Korean J Pain. 2018;31(2):109-15.

23. Marchesini M, Flaviano E, Bellini V, Baciarello M, Bignami E. Complication of epiduroscopy: a brief review and case report. Korean J Pain. 2018;31(4):296.

\section{Publisher's Note}

Springer Nature remains neutral with regard to jurisdictional claims in published maps and institutional affiliations.

\section{Submit your manuscript to a SpringerOpen ${ }^{\circ}$ journal and benefit from:}

- Convenient online submission

- Rigorous peer review

- Open access: articles freely available online

- High visibility within the field

- Retaining the copyright to your article

Submit your next manuscript at $\boldsymbol{\nabla}$ springeropen.com 